\title{
Cumulative beam breakup due to resistive-wall wake
}

\author{
Jiunn-Ming Wang* \\ National Synchrotron Light Source, Brookhaven National Laboratory, Upton, New York 11973, USA
}

Juhao $\mathrm{Wu}^{\dagger}$

Stanford Linear Accelerator Center, Stanford University, Stanford, California 94309, USA

(Received 20 June 2003; revised manuscript received 29 January 2004; published 25 March 2004)

\begin{abstract}
The cumulative beam breakup problem excited by the resistive-wall wake is formulated. An approximate analytic method of finding the asymptotic behavior for the transverse bunch displacement is developed and solved. Comparison between the asymptotic analytical expression and the direct numerical solution is presented. Good agreement is found. The criterion of using the asymptotic analytical expression is discussed.
\end{abstract}

DOI: 10.1103/PhysRevSTAB.7.034402

PACS numbers: 29.27.Bd, 52.35.Qz, 41.75.Ht, 07.85.Qe

\section{INTRODUCTION}

The cumulative beam breakup (BBU) problem in a periodic linear accelerator (linac) excited by the resonance wake is well understood [1-4]. However, there are no systematic studies for the corresponding problem excited by the resistive-wall impedance. This paper is a partial attempt to fill this gap. In a cylindrical circular metal pipe, if it is transversely offset, an electron beam will induce both asymmetric image charges and asymmetric image current in the metal wall. The electric field induced by this asymmetric image charge will attract the beam even closer to the metal wall, though the magnetic field induced by the asymmetric image current will push the beam back to the axis. The Lorentz forces due to the electric field and the magnetic field will partially cancel each other and result in a force which will attract the beam closer to the metal wall. The magnitude of this net force is proportional to $1 / \gamma^{2}$, with $\gamma$ to be the Lorentz relativistic energy factor. Hence for an ultrarelativistic electron beam, the instantaneous net force is negligible. However, the magnetic field will diffuse in the metal wall; therefore the magnetic force will decrease with time. On the other hand, since the electric field will not decay, the resulting net effect is a long-range wakefield [5]. In this paper, we study the BBU problem due to this long-range resistive-wall wakefield.

This study of the resistive-wall BBU problem is necessitated by the recently proposed Photoinjected Energy Recovering Linac (PERL) project [6]. For PERL, the light source consists of 12 undulators, each $12 \mathrm{~m}$ long, totaling $144 \mathrm{~m}$. The beam is shielded from the environment by circular copper pipes with a very small radius $b=3 \mathrm{~mm}$. The proposed injection cycle is $12 \mathrm{~h}$. It is crucial to know if the PERL beam can survive the BBU. We also investigate some other projects: the Linac Coherent

\footnotetext{
*Electronic address: jmwang@bnl.gov

${ }^{\dagger}$ Electronic address: jhwu@SLAC.Stanford.EDU
} ultraviolet (VUV) free-electron laser (FEL) at the Tera-electron-volt Energy Superconducting Linear Accelerator (TESLA) Test Facility (TTF) [8], the final focusing system (FFS) of the Next Linear Collider (NLC) [9], and the FFS of the TESLA [10]. For these cases, we study how the resistive-wall wake will dilute the beam.

The problem is formulated as follows: An electron bunch train consisting of a series of identical pointlike bunches passes through a cylindrical circular pipe of radius $b$ and conductivity $\sigma_{c}$. The entrance to the pipe is located at $z=0$, and the $M$ th bunch, $M=0,1,2, \ldots$, moves in the $z$ direction according to $z=c t-M c \tau_{B}$, where $\tau_{B}$ is the bunch separation in units of seconds. Inside the pipe, the equation of motion for a particle in bunch $M$ is

$$
\hat{O} y_{M} \equiv y_{M}^{\prime \prime}(z)+k_{y}^{2} y_{M}(z)=\sum_{N=0}^{M-1} S(M-N) y_{N}(z),
$$

where $y_{M}(z)$ is the transverse displace of the $M$ th bunch at location $z$, the prime stands for $d / d z$, and $k_{y}$ is the external focusing. The right-hand side of Eq. (1) represents the effects of the wake force. So far, we have not specified the wake yet; hence Eq. (1) is applicable to arbitrary wake for a beam with constant energy. In this paper, we will focus on the resistive-wall wake, which is [11]

$$
S(M)=a / \sqrt{M}
$$

with

$$
a=4 \frac{I_{B}}{I_{A}} \frac{1}{b^{3}} \delta_{\text {skin }},
$$

where $I_{B}=e N_{B} / \tau_{B}, \quad e N_{B}=$ bunch charge, $\quad I_{A} \equiv$ $4 \pi \epsilon_{0} m c^{3} \gamma / e=\gamma I_{\text {Alfvèn }}$ with $I_{\text {Alfvèn }} \approx 17000 \mathrm{~A}, m$ is the electron mass, $c$ is the speed of light in vacuum, $\epsilon_{0}=$ $8.854 \times 10^{-12} \mathrm{C}^{2} \mathrm{~N}^{-1} \mathrm{~m}^{-2}$ is the vacuum permittivity, and $\delta_{\text {skin }}=\sqrt{2 / \mu_{0} \sigma_{c} \omega_{\mathrm{B}}}=$ the skin depth corresponding 
to the bunch frequency $f_{B}=\omega_{B} / 2 \pi=1 / \tau_{B}$ with $\mu_{0}=$ $4 \pi \times 10^{-7} \mathrm{H} \mathrm{m}^{-1}$, the vacuum permeability. We ignore the effects of the wake force of a bunch on itself; as a consequence, the upper limit of the sum in Eq. (1) is $M-1$ instead of $M$. The single-bunch resistive-wall effect is studied separately $[12,13]$. The thickness of the beam pipe is assumed to be $\infty$ for convenience. Also notice that bunch $N$ is in front of bunch $M$ if $M>N$. In writing the above equations, we assumed the linac to be uniformly filled. For such a case, the locally averaged current $I_{\text {average }}=I_{B}$. For the case of nonuniform filling, an example of which will be discussed in Sec. V, Eq. (1) has to be modified.

The paper is organized as follows: In Sec. II, we set up the related eigenvalue problem of Eq. (1). Physically, the eigenfunction so obtained describe the beam coherent oscillation of an "extended problem" [14]. In Sec. III, we give a formal solution for the initial value problem. The solution consists of an integral representation for the transverse position of the $M$ th bunch at a longitudinal position $z$ in terms of the eigenfunctions obtained in the previous section [14]. The asymptotic limit, $M \rightarrow \infty$, of the transient solution is then obtained in Sec. IV for two extreme cases: the no focusing (NF) case and the strong focusing (SF) case. In Secs. II, III, IV, and V, we treat the case where only one bunch is offset initially. While in Secs. II, III, and IV, we treat the case where every bucket of the linac is filled by the same amount of charge, we treat in Sec. V the case where the filling pattern is such that the beam has periodically unfilled gaps. The results of Sec. V are compared to the results of the preceding sections. The conclusion we draw from the comparison is that the asymptotic resistive-wall cumulative BBU is a locally averaged current problem. In Sec. VI we go back to the problem where each bucket is uniformly filled. The difference between this section and Sec. IV is that here we treat the case where initially the transverse position of every bunch is offset by the same amount-injection error. By comparing the results of Sec. VI with those of Sec. IV, we observe a screening effect for the injection error case. The analytical asymptotic solutions are checked against the directly numerical solution in Sec. VII. Good agreement between the asymptotic analytical expression and the direct numerical solution is found. We then apply the analytical results to the PERL project, the final undulator of LCLS and TTF VUV FEL, and also the FFS of the NLC and TESLA in Sec. VIII. Discussion and conclusion are also presented there.

\section{THE EIGENVALUE PROBLEM}

In this section, let us formulate the eigenvalue problem of Eq. (1). The right-hand side of Eq. (1) is a convolution sum, therefore, it can be diagonalized by a Fourier transform. Define

$$
F(\theta)=\sum_{M=1}^{\infty} \frac{1}{\sqrt{M}} e^{i M \theta}
$$

and

$$
\xi(\theta, z)=\sum_{M=0}^{\infty} y_{M}(z) e^{i M \theta}
$$

then

$$
y_{M}(z)=\frac{1}{2 \pi} \int_{-\pi}^{\pi} d \theta e^{-i M \theta} \xi(\theta, z)
$$

and

$$
\xi^{\prime \prime}(\theta, z)+k_{y}^{2} \xi(\theta, z)=a F(\theta) \xi(\theta, z) .
$$

The last equation is an eigenvalue equation, with the parameter $\theta$ playing the role of distinguishing different eigenvalues. For the coherent mode $\theta$, we see from Eq. (5) that the parameter $\theta$ is the phase difference of the adjacent bunches in this mode. Recall that in a storage ring, a symmetric coupling bunch mode $n$ is characterized by the Courant-Sessler phase factor $\exp (i 2 \pi n / h)$ [15], where $h$ is the number of bunches in the ring. We can think of the phase $\exp (i \theta)$ as the limit of the Courant-Sessler factor when both $n$ and $h \rightarrow \infty$, while $2 \pi n / h=\theta$ remains finite. The eigenvalue for the mode $\theta$ is, from Eq. (7),

$$
k_{c}(\theta)=\sqrt{k_{y}^{2}-a F(\theta)},
$$

and the corresponding eigenvectors are

$$
\cos \left[k_{c}(\theta) z\right] \text { or } \sin \left[k_{c}(\theta) z\right] .
$$

The function $F(\theta)$ can be written as [16]

$$
\begin{aligned}
F(\theta) & =\sqrt{\frac{i \pi}{\theta}}+\sum_{n=0}^{\infty} \zeta_{\text {Riemann }}\left(\frac{1}{2}-n\right) \frac{(i \theta)^{n}}{n !} \\
& \approx \sqrt{\frac{i \pi}{\theta}}-1.460-0.208 i \theta+\mathcal{O}\left(\theta^{2}\right),
\end{aligned}
$$

where $\zeta_{\text {Riemann }}(x)$ is the Riemann's zeta function. The function $F(\theta)$ has a branch point at $\theta=0$, therefore, through Eq. (7), $\xi(\theta, z)$ also has a singular point at the same position. Since Eq. (6) is the inverse of Eq. (5) and we look for $y_{M}$ with $M>0$, causality requires this singularity to lie below the contour of Eq. (6) on the $\theta$ plane. In order to explain this point more clearly, let us introduce

$$
\zeta \equiv e^{i \theta}
$$

In terms of this variable, Eqs. (4)-(7) become

$$
\begin{gathered}
F(\zeta)=\sum_{M=1}^{\infty} \frac{1}{\sqrt{M}} \zeta^{M}, \\
\xi(\zeta, z)=\sum_{M=0}^{\infty} y_{M}(z) \zeta^{M},
\end{gathered}
$$




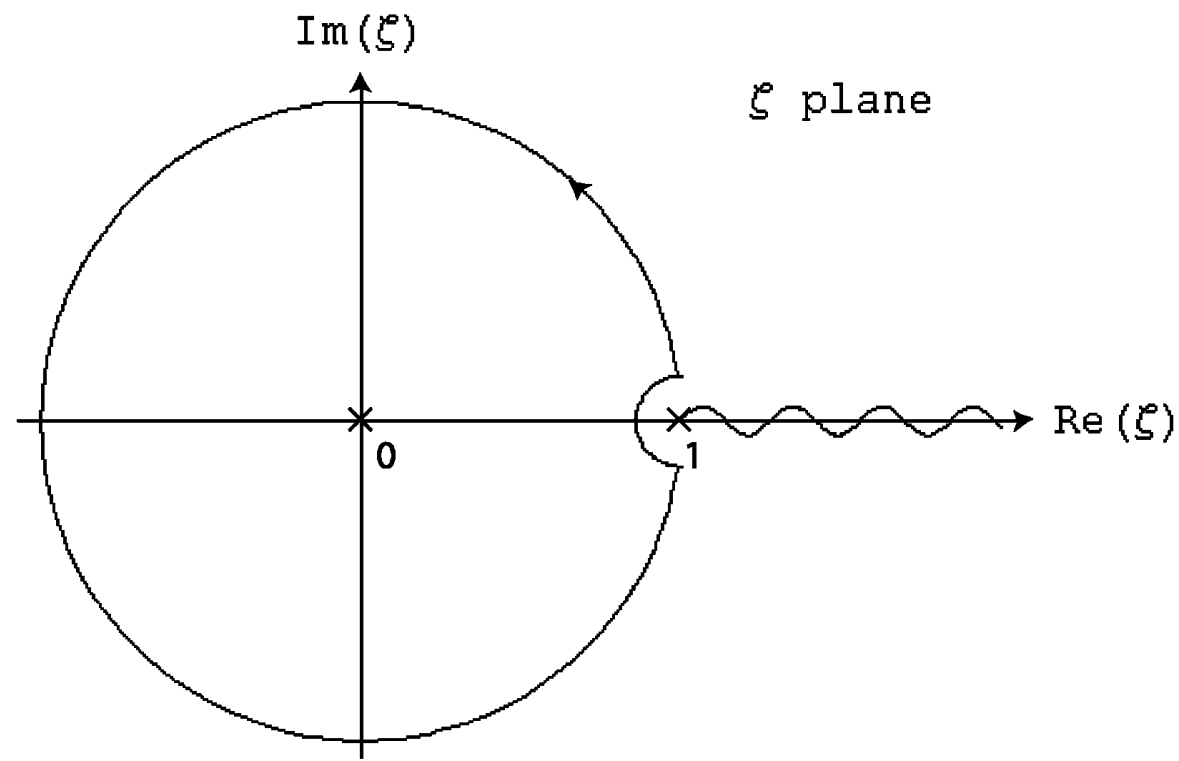

FIG. 1. The contour used for the definition in Eq. (14).

$$
y_{M}(z)=\frac{1}{2 \pi i} \oint d \zeta \zeta^{-(M+1)} \xi(\zeta, z)
$$

and

$$
\xi^{\prime \prime}(\zeta, z)+k_{y}^{2} \xi(\zeta, z)=a F(\zeta) \xi(\zeta, z)
$$

When expressing a function of $\theta$, for example, the function $F(\theta)$, in terms of $\zeta$, we write $F(\zeta)=F(\theta)$ instead of creating a new symbol; this should not introduce any unnecessary confusion. We adopt this convention throughout this paper. The singularity of $F(\theta)$ at $\theta=0$ corresponds to a singularity of $F(\zeta)$ at $\zeta=1$. The singular part of $F(\zeta)$ is

$$
F(\zeta) \cong \sqrt{\frac{\pi}{1-\zeta}} \text { for } \zeta \rightarrow 1
$$

Equation (13) is a power series expansion of the function $\xi$ in the variable $\zeta$. The radius of the convergence circle of this series is 1 , since the closest singularity of $\xi$ is at $\zeta=1$, i.e., at $\theta=0$. From the residue theorem, Eq. (14) is clearly the inverse of Eq. (13) provided that the integration contour lies inside of the convergence circle, and the contour encircles the origin $\zeta=0$ counterclockwise. The contour is shown in Fig. 1. It is convenient to deform the contour to be the unit circle and place the singularity at $\zeta=1+\epsilon$ with a small and positive $\epsilon$. On the $\zeta$ plane, we make a cut on the real axis from $\zeta=$ $1+\epsilon$ to $\zeta=\infty$, and make all the following calculations on the first sheet of the Riemann surface. In terms of the $\theta$ variable, the singularity is at $\theta=-i \log (1+\epsilon) \approx-i \epsilon$, i.e., below the contour of Eq. (6). The cut on the $\theta$ plane is at the lower half of the imaginary axis, i.e., $\theta$ from $-i \epsilon$ to $-i \infty$.
We solve in the next section the transient BBU problem by relating it to the coherent solutions given by Eqs. (8) and (9).

\section{INITIAL VALUE PROBLEM}

One can carry out the BBU calculations in terms of either the $\zeta$ or the $\theta$ variable. We choose to use the variable $\zeta$.

We show in this section that the transient solution to the equation of motion (1) is

$$
\begin{aligned}
y_{M}(z)= & y_{M 0} \cos \left(k_{y} z\right)+y_{M 0}^{\prime} \sin \left(k_{y} z\right) / k_{y} \\
& +\frac{1}{2 \pi i} \sum_{N=0}^{M-1} y_{N 0} \oint d \zeta \zeta^{-(M-N+1)} \cos \left[k_{c}(\zeta) z\right] \\
& +\frac{1}{2 \pi i} \sum_{N=0}^{M-1} y_{N 0}^{\prime} \oint d \zeta \zeta^{-(M-N+1)} \frac{\sin \left[k_{c}(\zeta) z\right]}{k_{c}(\zeta)},
\end{aligned}
$$

where $y_{M 0}$ and $y_{M 0}^{\prime}$ are, respectively, the initial values (values at $z=0$ ) of $y_{M}(z)$ and $y_{M}^{\prime}(z)$.

First, we find the transient solution of Eq. (15). This equation yields

$$
\tilde{\xi}(\zeta, s)=\frac{s \xi(\zeta, 0)+\xi^{\prime}(\zeta, 0)}{s^{2}+k_{y}^{2}-a F(\zeta)},
$$

where

$$
\tilde{\xi}(\zeta, s) \equiv \int_{0}^{\infty} d z \xi(\zeta, z) e^{-s z} .
$$

After carrying out the inverse Laplace transform of (19), using (18), we obtain

$$
\xi(\zeta, z)=\xi(\zeta, 0) \cos \left[k_{c}(\zeta) z\right]+\xi^{\prime}(\zeta, 0) \frac{\sin \left[k_{c}(\zeta) z\right]}{k_{c}(\zeta)} .
$$


In order to obtain (17), we substitute the above result (20) into (14) and then use $\xi(\zeta, 0)=\sum_{M=0}^{\infty} y_{M, 0} \zeta^{M}$ and $\xi^{\prime}(\zeta, 0)=\sum_{M=0}^{\infty} y_{M, 0}^{\prime} \zeta^{M}$. The result is (17). We shall now apply the solution (17) to some specific cases in the next section.

\section{INITIAL SINGLE-BUNCH OFFSET}

We study in this section Eq. (17) for the case where only the first bunch $M=0$ is initially offset transversely from the center of the chamber, $y_{M 0}=y_{00} \delta_{M, 0}$, and $y_{M 0}^{\prime}=0, \quad \forall M$. Then, Eq. (17) becomes, for $M \neq 0$,

$$
\begin{aligned}
y_{M}(z) & =\frac{1}{2 \pi i} y_{00} \oint d \zeta \zeta^{-(M+1)} \cos \left[k_{c}(\zeta) z\right] \\
& \equiv \frac{y_{00}}{4 \pi}\left[\eta_{M}^{(+)}(z)+\eta_{M}^{(-)}(z)\right],
\end{aligned}
$$

where

$$
\eta_{M}^{( \pm)}(z) \equiv \frac{1}{i} \oint d \zeta \exp \left\{\Psi_{M}^{( \pm)}(\zeta)\right\}
$$

with

$$
\Psi_{M}^{( \pm)}(\zeta)= \pm i k_{c}(\zeta) z-(M+1) \log (\zeta)
$$

We wish to find the asymptotic behavior of $y_{M}$ as given by Eq. (21) when $M \rightarrow \infty$; we shall use the well-known saddle point method for this purpose.

The asymptotic behavior of the integral (21) is determined by the behavior of $\cos \left[k_{c}(\zeta) z\right]$ near $\zeta=1$ or $\theta=0$, where the phase difference between adjacent bunches approaches zero. In other words, the saddle point $\zeta_{\text {saddle }} \rightarrow 1$, or equivalently, $\theta_{\text {saddle }} \rightarrow 0$ in the limit of $M \rightarrow \infty$. The behavior of $\cos \left[k_{c}(\zeta) z\right]$ near $\zeta=1$ is, from Eq. (8), controlled by the behavior of $F(\zeta)$ in the same neighborhood. We shall use the approximation Eq. (16) for $F(\zeta)$ throughout the rest of this paper. Combining the expression (16) with Eqs. (8) and (15), we have

$$
k_{c}(\zeta)=\sqrt{k_{y}^{2}-a \sqrt{\pi /(1-\bar{\zeta})}}
$$

and

$$
\xi^{\prime \prime}(\zeta, z)+k_{y}^{2} \xi(\zeta, z)=a \sqrt{\frac{\pi}{1-\zeta}} \xi(\zeta, z)
$$

The last equation together with Eq. (14) makes up the basis for the remainder of this section.

We shall carry out below the asymptotic analysis of the following two cases:

First case: This is the case where either $k_{y}=0$, or $M$ is so large that the $a \sqrt{\pi /(1-\zeta)}$ term dominates over $k_{y}^{2}$ in Eq. (24). As a consequence, we can use the approximate expression

$$
k_{c}(\zeta)=a_{1} i(1-\zeta)^{-1 / 4},
$$

where $a_{1}=\sqrt{a \sqrt{\pi}}$. This case will be referred to as the no focusing case. Clearly, in order for this approximation to be valid, the condition $\left|a_{1}\left(1-\zeta_{\mathrm{NF}}\right)^{-1 / 4}\right| \gg k_{y}$, has to be satisfied, where $\zeta_{\mathrm{NF}}$ is the saddle point.

Second case: This is the case where $M$ is so large that Eq. (16) is valid, and yet $k_{y}^{2}$ in Eq. (24) dominates over the $a \sqrt{\pi /(1-\zeta)}$ term. As a consequence,

$$
k_{c}(\zeta) \cong k_{y}-2 a_{2}(1-\zeta)^{-1 / 2},
$$

where $a_{2}=a \sqrt{\pi} /\left(4 k_{y}\right)$. We shall refer to this case as the strong focusing case. The condition for the validity of this approximation is $k_{y} \gg\left|a_{1}\left(1-\zeta_{\mathrm{SF}}\right)^{-1 / 4}\right|$, where $\zeta_{\mathrm{SF}}$ is the saddle point.

The remainder of this section is devoted to the detailed treatment of these two cases. Some of the results in this section have been briefly reported in Ref. [17].

\section{A. No focusing case}

We wish to carry out the saddle point analysis to the integrals (21) and (22) with

$$
\begin{gathered}
\Psi_{M}^{( \pm)}(\zeta)=(M+1)\left[\mp 4 \alpha_{1}(1-\zeta)^{-1 / 4}-\log (\zeta)\right], \\
\dot{\Psi}_{M}^{( \pm)}(\zeta)=(M+1)\left[ \pm \alpha_{1}(1-\zeta)^{-5 / 4}-1 / \zeta\right],
\end{gathered}
$$

and

$$
\ddot{\Psi}_{M}^{( \pm)}(\zeta)=(M+1)\left[\mp(5 / 4) \alpha_{1}(1-\zeta)^{-9 / 4}+1 / \zeta^{2}\right],
$$

where "." stands for $d / d \zeta$, and $\alpha_{1} \equiv a_{1} z /[4(M+1)]$. The function $\Psi_{M}^{( \pm)}(\zeta)$ has branch points at $\zeta=0$ and $\zeta=$ 1. Let us draw cuts in the $\zeta$ plane from $\zeta=-\infty$ to 0 , and from $\zeta=1$ to $\infty$. The integral (21) is performed on the first sheet of $\Psi_{M}^{(\dot{ \pm})}(\zeta)$ which is defined to be the sheet where $\Psi_{M}^{( \pm)}(\zeta)=$ real for $0<\zeta<1$. The contour of the saddle point integral is shown in Fig. 2.

The saddle point $\zeta_{\mathrm{NF}}$ satisfies $\dot{\Psi}_{M}^{( \pm)}\left(\zeta_{\mathrm{NF}}\right)=0$, or

$$
\left(1-\zeta_{\mathrm{NF}}\right)^{5 / 4}= \pm \alpha_{1} \zeta_{\mathrm{NF}}
$$

This equation cannot be solved algebraically. However noting that $\alpha_{1}=\mathcal{O}(1 / M)$ is small in the limit of $M \rightarrow \infty$, we solve the equation by perturbation. In terms of the variable $\tilde{\zeta} \equiv 1-\zeta$, Eq. (31) becomes, to the lowest order in $\alpha_{1}$,

$$
\tilde{\zeta}_{\mathrm{NF}}^{5 / 4}=\mp \alpha_{1} .
$$

Taking the fourth power of this equation, we have

$$
\tilde{\zeta}_{\mathrm{NF}}^{5}=\alpha_{1}^{4},
$$

yielding the solutions

$$
\zeta_{\mathrm{NF}}=\left(1-\alpha_{1}^{4 / 5}, 1-\alpha_{1}^{4 / 5} e^{ \pm i 2 \pi / 5}, 1-\alpha_{1}^{4 / 5} e^{ \pm i 4 \pi / 5}\right),
$$

where we write the five solutions of Eq. (33) as a compact 


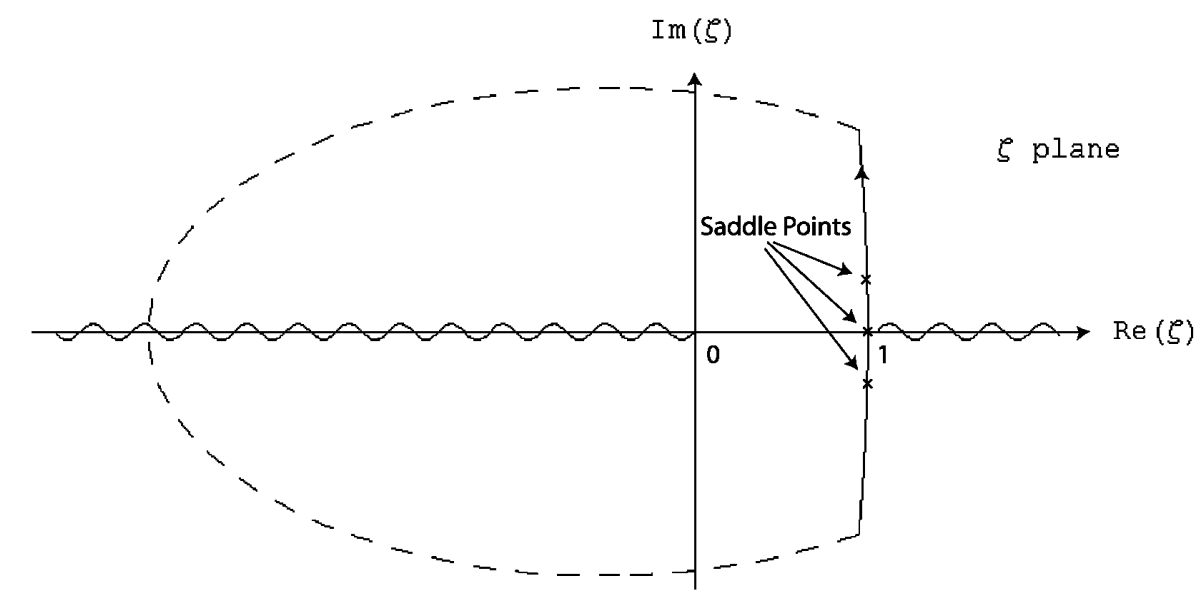

FIG. 2. The contour used for the saddle point calculation. The dashed curve indicates that the contour is closed at $|\zeta| \rightarrow \infty$.

row matrix. The condition (33) is a necessary but not a sufficient condition for saddle points. [We took the fourth power of Eq. (32) in order to obtain Eq. (33); we might in doing so have introduced spurious solutions.] Each of the solutions (34) has yet to be verified to be a relevant saddle point. It is straightforward to verify that $\zeta_{\mathrm{NF}}^{(-)}=1-\alpha_{1}^{4 / 5}$ is the only saddle point of $\eta^{(-)}$and $\zeta_{\mathrm{NF}}^{(+)}=1-\alpha_{1}^{4 / 5} e^{ \pm i 4 \pi / 5}$ are the only saddle points of $\eta^{(+)}$we have to consider. These saddle points are also indicated in Fig. 2.

The saddle point contribution to $\eta^{( \pm)}$satisfies

$$
\eta_{M}^{( \pm)} \propto \exp \left[\Psi^{( \pm)}\left(\zeta_{\mathrm{NF}}^{( \pm)}\right)\right] .
$$

Routine calculation gives the following results for the exponents:

$$
\Psi_{M}^{(-)}\left(\zeta_{\mathrm{NF}}^{(-)}\right)=5(M+1) \alpha_{1}^{4 / 5}
$$

and

$$
\Psi_{M}^{(+)}\left(\zeta_{\mathrm{NF}}^{(+)}\right)=5(M+1) \alpha_{1}^{4 / 5} \exp ( \pm i 4 \pi / 5) .
$$

Notice that the real part of $\Psi_{M}^{(+)}$above is negative; therefore, $\eta_{M}^{(+)} \rightarrow 0$ in the limit of $M \rightarrow \infty$. We shall ignore the $\eta_{M}^{(+)}$term in Eq. (21).

In order to perform the saddle point integral for $\eta_{M}^{(-)}$ we need, in addition to (36), the following expression:

$$
\ddot{\Psi}_{M}^{(-)}\left(\zeta_{\mathrm{NF}}^{(-)}\right)=\frac{5(M+1)}{4 \alpha_{1}^{4 / 5}}
$$

We notice that $\ddot{\Psi}_{M}^{(-)}\left[\zeta_{\mathrm{NF}}^{(-)}\right] \propto \alpha_{1}^{-9 / 5} \propto M^{9 / 5} \rightarrow \infty$ rapidly, as $M \rightarrow \infty$. Such sharp dependence of the integrand of (22) in the neighborhood of the saddle point validates the saddle point approximation.

From the above discussion, the equation

$$
y_{M}(z)=\frac{y_{00}}{4 \pi} \eta_{M}^{(-)}(z) \propto \exp \left[\Psi_{M}^{(-)}\left(\zeta_{\mathrm{NF}}^{(-)}\right)\right]
$$

together with Eqs. (36) and (38) are all we need for the saddle point estimate of the present BBU problem. However, before stating the results, let us have a discussion on the growth time $t_{\mathrm{NF}}$ of the mode under discussion.

The $M$ th bunch reaches the linac at time $t=M \tau_{B}$. The quantity $\alpha_{1}$ in the expression (36) can be written in terms of $M$ and $a$. If we replace $M$ or $M+1$ (recall that $M \gg 1$ ) in the resulting $\Psi_{M}^{(-)}$by $t / \tau_{B}$, we obtain

$$
\Psi_{M}^{(-)}\left(\zeta_{\mathrm{NF}}^{(-)}\right)=\left(\frac{t}{t_{\mathrm{NF}}}\right)^{1 / 5},
$$

where the growth time

$$
t_{\mathrm{NF}}=\frac{\tau_{B}}{4 \pi}\left(\frac{4}{5}\right)^{5} \frac{1}{z^{4}} \frac{1}{a^{2}},
$$

and the result of the saddle point integral is

$y_{M}(z)=\frac{y_{00}}{4 \pi} \eta_{M}^{(-)}(z)=\frac{y_{00}}{5 \sqrt{2 \pi}}\left(\frac{t_{\mathrm{NF}}}{t}\right)^{9 / 10} \frac{\tau_{B}}{t_{\mathrm{NF}}} \exp \left\{\left(\frac{t}{t_{\mathrm{NF}}}\right)^{1 / 5}\right\}$.

So far we have been dealing with the case of a uniformly filled linac. If the filling is not uniform (some buckets not filled) the above results do not hold. In Sec. V, we shall treat an example of such a nonuniform case. In order to facilitate later comparison, let us write Eq. (41) for $t_{\mathrm{NF}}$ in another form. Using Eq. (3), Eq. (41) becomes

$$
t_{\mathrm{NF}}=\frac{\tau_{B}}{\pi} \frac{16}{5^{5}} \frac{b^{6}}{z^{4}} \frac{1}{\delta_{\text {skin }}^{2}} \frac{I_{A}^{2}}{I_{B}^{2}} .
$$

For the case of uniform filling, the $I_{B}=e N_{B} / \tau_{B}$ above equals the locally averaged current $I_{\text {average }}$. Therefore the above equation can be expressed as

$$
t_{\mathrm{NF}}=\frac{\tau_{B}}{\pi} \frac{16}{5^{5}} \frac{b^{6}}{z^{4}} \frac{1}{\delta_{\text {skin }}^{2}} \frac{I_{A}^{2}}{I_{\text {average }}^{2}} .
$$

We shall compare later the above expressions (43) and (44) with the corresponding results for a nonuniformly filled beam. 


\section{B. Strong focusing case}

The treatment of this case is similar to the NF case. Having given all the calculation details for the NF case, here we present the results directly. The asymptotic result for the displacement of the $M$ th bunch is

$$
\begin{aligned}
y_{M}(z)=\frac{2 y_{00}}{3 \sqrt{2 \pi}}\left(\frac{t_{\mathrm{SF}}}{t}\right)^{5 / 6} \frac{\tau_{B}}{t_{\mathrm{SF}}} \exp \left\{\left(\frac{t}{t_{\mathrm{SF}}}\right)^{1 / 3}\right\} \cos [ & \sqrt{3}\left(\frac{t}{t_{\mathrm{SF}}}\right)^{1 / 3} \\
& \left.-k_{y} z+\frac{\pi}{6}\right],
\end{aligned}
$$

where the growth time for this mode is

$$
t_{\mathrm{SF}} \equiv \tau_{B}\left(\frac{2}{3}\right)^{3} \frac{1}{a_{2}^{2} z^{2}}
$$

and again $t=(M+1) \tau_{B}$, or $M \tau_{B}$ since $M$ is large. For readers who are interested in the calculation details, please refer to Appendix A.

\section{BEAM WITH PERIODIC GAPS}

The bunch filling pattern considered in this section is as follows: The beam is made of repetitive identical sequences where each sequence consists of $p$ adjacent filled buckets followed by $q$ empty buckets; there are in total $r=p+q$ buckets in a sequence.

\section{A. Equations of motion}

If all the buckets are filled, then the equation of motion is given by Eq. (1), i.e.,

$$
\hat{O} y_{M} \equiv\left(\frac{d^{2}}{d z^{2}}+k_{y}^{2}\right) y_{M}=\sum_{N=1}^{M-1} S(M-N) y_{N},
$$

where $S(M)$ is the wake function given in Sec. I; $S(M)=$ 0 for $M \leq 0$, and $S(M-N)=a / \sqrt{M-N}$ for $M>0$. The parameter $a$ is given by Eq. (3). Note that we have made here a slight change of convention. We designated the bunches as $M=1,2,3, \ldots$ above instead of $M=$ $0,1,2, \ldots$ as was done in Sec. I. We adopt this new convention throughout this section.

We have to generalize the above equation to include the case of a beam with periodic empty buckets. Let us use the notation $u=1,2,3, \ldots$ for the sequence number, and $m=1,2, \ldots, p$ for the bunch number in a sequence. It is convenient to define, corresponding to each $u$, a $p \times p$ matrix $\mathbb{S}^{(u)}$ with its elements given by

$$
\mathbb{S}_{m, n}^{(u)}=\mathbb{S}_{m-n}^{(u)}=S[u r+(m-n)],
$$

where the range of $u$ for $\mathbb{S}^{(u)}$ is $u=0,1,2, \ldots$ Corresponding to the above matrix, we define $1 \times p$ column vector

$$
Y^{(u)} \equiv\left(\begin{array}{c}
y_{u, 1} \\
y_{u, 2} \\
\vdots \\
y_{u, p}
\end{array}\right),
$$

where $y_{u, m}$ is the transverse displacement of the $m$ th bunch in the $u$ th sequence.

The equation of motion for a beam with periodic gaps can now be written in a compact form similar to Eq. (1),

$$
\hat{O} Y^{(u)}=\sum_{v=1}^{u} \mathbb{S}^{(u-v)} Y^{(v)}
$$

We solve this equation in the next subsection.

\section{B. Solutions}

The $m$ th component of the equation of motion (49) is

$$
\hat{O} y_{u, m}=\sum_{v=1}^{u} \sum_{n=1}^{p} \mathbb{S}_{m-n}^{(u-v)} y_{v, n} .
$$

The following generalization of Eqs. (12) and (13) is convenient:

$$
\begin{aligned}
& \xi_{m}(\zeta)=\sum_{u=1}^{\infty} \zeta^{u} y_{u, m}, \\
& \Delta_{m}(\zeta)=\sum_{u=0}^{\infty} \mathbb{S}_{m}^{(u)} \zeta^{u} .
\end{aligned}
$$

Then the above three Eqs. (50)-(52) lead to

$$
\hat{O} \xi_{m}(\zeta)=\sum_{n=1}^{p} \Delta_{m-n}(\zeta) \xi_{n}(\zeta)
$$

Once the solution of the last equation is found, the displacement of the individual bunch is found by substituting the solution into the inverse of (51), namely,

$$
y_{u, m}=\frac{1}{2 \pi i} \oint d \zeta \zeta^{-(u+1)} \xi_{m}(\zeta)
$$

The method we use to solve Eq. (53) is a generalization of the method of Sec. IV. Note that for $u \rightarrow \infty$, the contribution to the integral (54) is dominated by the behavior of the integrand near $\zeta=1$. Therefore we shall, in analogy to what we did in Sec. IV, approximate $\Delta_{m}$ by its singular part near $\zeta=1$. The singular part is from [16]

$$
\Delta_{m}(\zeta) \cong \frac{a}{\sqrt{r}} \sqrt{\frac{\pi}{1-\zeta}} \quad \forall m,
$$

and the corresponding approximation to Eq. (53) is

$$
\hat{O} \xi_{m}(\zeta) \cong \frac{a p}{\sqrt{r}} \sqrt{\frac{\pi}{1-\zeta}} \xi_{m}(\zeta), \quad \forall m .
$$


This equation together with Eq. (54) gives us the asymptotic behavior, $u \rightarrow \infty$, of $y_{u, m}$.

Observe the similarity of Eqs. (54) and (56) above to the following equations we obtained earlier for the uniform filling case, i.e., Eqs. (14) and (25):

$$
\begin{aligned}
\hat{O} \xi(\zeta, z) & =a \sqrt{\frac{\bar{\pi}}{1-\zeta}} \xi(\zeta, z), \\
y_{M}(z) & =\frac{1}{2 \pi i} \oint d \zeta \zeta^{-(M+1)} \xi(\zeta, z) .
\end{aligned}
$$

The variable $m$ appears as a passive parameter in Eqs. (54) and (56). Also, these equations can be obtained from Eqs. (14) and (25) by the following substitutions:

$$
\begin{gathered}
M, \quad \text { or } \quad(M+1) \rightarrow u, \\
a \rightarrow a p / \sqrt{r} .
\end{gathered}
$$

Therefore, we can obtain the results for Eqs. (54) and (56) from the corresponding results for the uniform filling case. We treat here the NF case corresponding to Sec. IVA. We specifically consider the growth time $t_{\mathrm{NF}}^{\text {gap }}$ for the beam with periodic gaps. The SF case can be treated in a similar way.

We start from the exponent $\Psi_{M}^{(-)}$as given by (36). Expressing $\alpha_{1}$ in terms of $a$, this equation is equivalent to

$$
\Psi_{M}^{(-)}\left(\zeta_{\mathrm{NF}}^{(-)}\right)=5 \pi^{1 / 5}(M+1)^{1 / 5}(z / 4)^{4 / 5} a^{2 / 5} .
$$

Now applying the substitution rules (57) and (58) to Eq. (59), we obtain

$$
\Psi_{\mathrm{gap}, u}^{(-)}\left(\zeta_{\mathrm{NF}}^{(-)}\right)=\left(\frac{u}{u_{\mathrm{NF}}}\right)^{1 / 5}
$$

where

$$
\begin{aligned}
y_{M}(z) & =y_{00} \cos \left(k_{y} z\right)-\frac{1}{2 \pi i} y_{00} \oint d \zeta \zeta^{-1}(1-\zeta)^{-1} \cos \left[k_{c}(\zeta) z\right]+\frac{1}{2 \pi i} y_{00} \oint d \zeta \zeta^{-(M+1)}(1-\zeta)^{-1} \cos \left[k_{c}(\zeta) z\right] \\
& =y_{00} \cos \left(k_{y} z\right)-y_{00} \cos \left[k_{c}(0) z\right]+\frac{y_{00}}{4 \pi}\left[\eta_{M}^{(+)}(z)+\eta_{M}^{(-)}(z)\right],
\end{aligned}
$$

where $\eta_{M}^{( \pm)}(z)$ is given by Eq. (22) with

$$
\Psi_{M}^{( \pm)}(\zeta)= \pm i k_{c}(\zeta) z-(M+1) \log (\zeta)-\log (1-\zeta) .
$$

Compared with Eq. (23), Eq. (68) has an additional term $-\log (1-\zeta)$ on the right-hand side. We shall see presently that this term does not change the eigensolutions as given in Secs. II, III, IV, and V, but it will change the transient solutions. We shall also see that this term leads to an interesting screening effect. From $k_{c}(0)=k_{y}$, Eq. (67) simplifies to

$$
y_{M}(z)=\frac{y_{00}}{4 \pi}\left[\eta_{M}^{(+)}(z)+\eta_{M}^{(-)}(z)\right]
$$

$$
u_{\mathrm{NF}}=\frac{1}{4 \pi}\left(\frac{4}{5}\right)^{5} \frac{1}{z^{4}} \frac{r}{a^{2} p^{2}}
$$

is the growth time in units of sequences.

We have to translate $u$ into time $t$. The bunch $(u, m)$ reaches the linac at $t=(u r+m) \tau_{B} \cong u r \tau_{B}$. Therefore we should set $u \rightarrow t / r \tau_{B}$ and

$$
\begin{aligned}
t_{\mathrm{NF}}^{\mathrm{gap}} & =r \tau_{B} u_{\mathrm{NF}} \\
& =\frac{\tau_{B}}{4 \pi}\left(\frac{4}{5}\right)^{5} \frac{1}{z^{4}} \frac{1}{a^{2}} \frac{r^{2}}{p^{2}} \\
& =\frac{\tau_{B}}{\pi} \frac{16}{5^{5}} \frac{b^{6}}{z^{4}} \frac{1}{\delta_{\text {skin }}^{2}} \frac{I_{A}^{2}}{I_{B}^{2}} \frac{r^{2}}{p^{2}} .
\end{aligned}
$$

This expression differs from Eq. (41) or (43) by a factor of $r^{2} / p^{2}$. However, this difference is superficial. Let us calculate the average current of a sequence. It is clearly

$$
I_{\text {average }}=\frac{p}{r} I_{B} .
$$

In terms of $I_{\text {average }}$, the growth time becomes

$$
t_{\mathrm{NF}}^{\mathrm{gap}}=\frac{\tau_{B}}{\pi} \frac{16}{5^{5}} \frac{b^{6}}{z^{4}} \frac{1}{\delta_{\text {skin }}^{2}} \frac{I_{A}^{2}}{I_{\text {average }}^{2}} .
$$

This is identical to Eq. (44). We therefore conclude that the cumulative resistive-wall BBU is a locally averaged current effect.

\section{INJECTION ERROR AND SCREENING EFFECT}

In this section, we study Eq. (17) for the case where BBU is started up by an injection error, i.e., all the bunches are initially offset by the same amount, $y_{M 0}=$ $y_{00}$, and $y_{M 0}^{\prime}=0, \quad \forall M \geq 0$. Then, Eq. (17) becomes 


$$
t_{\mathrm{NF}}=\frac{\tau_{B}}{4 \pi}\left(\frac{4}{5}\right)^{5} \frac{1}{z^{4}} \frac{1}{a^{2}}
$$

and

$$
G_{\mathrm{NF}} \equiv 5\left(\frac{t}{\tau_{B}}\right)^{4 / 5}\left(\frac{t_{\mathrm{NF}}}{\tau_{B}}\right)^{1 / 5}=4\left(\frac{1}{4 \pi a^{2}}\right)^{1 / 5}\left(\frac{M}{z}\right)^{4 / 5}
$$

The calculation details can be found in Appendix B. It is very interesting to compare the above result (69) to the result (42) of the initial single-bunch offset case. We find the following: (i) The growth time $t_{\mathrm{NF}}$ is the same for both cases, as it should be, since $t_{\mathrm{NF}}$ should depend only on the eigensolutions; (ii) the only difference between the transient solutions is the factor $G_{\mathrm{NF}}$ which is proportional to $M^{4 / 5}$ instead of to $M$ (recall that $t \propto M$ ). This is surprising: Since $\theta_{\text {saddle }} \cong 0$, we would expect all the bunches preceding the bunch $M$ to excite this bunch by the same amount leading to $G_{\mathrm{NF}} \propto M$. Clearly, the preceding bunches are screening each other. (It can actually be shown that for large but not too large $M$, the function $\left.G_{\mathrm{NF}} \propto M.\right)$

\section{B. Strong focusing case}

Similar to those of Secs. IV and VI A, we find the asymptotic result for the SF case as

$$
\begin{aligned}
y_{M}(z)= & G_{\mathrm{SF}} \frac{2 y_{00}}{3 \sqrt{2 \pi}}\left(\frac{t_{\mathrm{SF}}}{t}\right)^{5 / 6} \frac{\tau_{B}}{t_{\mathrm{SF}}} \exp \left\{\left(\frac{t}{t_{\mathrm{SF}}}\right)^{1 / 3}\right\} \\
& \times \cos \left[\sqrt{3}\left(\frac{t}{t_{\mathrm{SF}}}\right)^{1 / 3}-k_{y} z-\frac{\pi}{6}\right],
\end{aligned}
$$

where $t=M \tau_{B}$, the growth time is again the same as what was given in Eq. (46), i.e.,

$$
t_{\mathrm{SF}} \equiv \tau_{B}\left(\frac{2}{3}\right)^{3} \frac{1}{a_{2}^{2} z^{2}}
$$

and

$$
G_{\mathrm{SF}} \equiv \frac{3}{2}\left(\frac{t}{\tau_{B}}\right)^{2 / 3}\left(\frac{t_{\mathrm{SF}}}{\tau_{B}}\right)^{1 / 3}=\left(\frac{16 k_{y}^{2}}{\pi a^{2}}\right)^{1 / 3}\left(\frac{M}{z}\right)^{2 / 3} .
$$

It is worth noting that, besides the overall factor $\mathcal{G}_{\mathrm{SF}}$, there is also an overall phase shift of $-\pi / 3$ between the above solution in Eq. (71) and that given by Eq. (45).

\section{COMPARISON WITH NUMERICAL RESULTS}

To check the analytical results, we compare them with direct numerical results. As what we comment after Eq. (66), the results for beams with periodic gaps in Sec. V can be obtained by proper variable transformation given in Eqs. (57) and (58). We will check only the results for the case of the initial single-bunch offset in Sec. IV and the case of the injection error in Sec. VI.

\section{A. Initial single-bunch offset}

The general solution is given in Eq. (21), i.e., for $M \neq 0$,

$$
\begin{aligned}
y_{M}(z) & =\frac{1}{2 \pi i} y_{00} \oint d \zeta \zeta^{-(M+1)} \cos \left[k_{c}(\zeta) z\right] \\
& \equiv \frac{y_{00}}{4 \pi}\left[\eta_{M}^{(+)}(z)+\eta_{M}^{(-)}(z)\right]
\end{aligned}
$$

This time we write

$$
\eta_{M}^{( \pm)}(z) \equiv \frac{1}{i} \oint d \zeta \zeta^{-(M+1)} \exp \left\{ \pm i k_{c}(\zeta) z\right\},
$$

where the contour is shown in Fig. 1, as the unit circle in the $\zeta$ plane and the singularity to be located at $\zeta=1+\epsilon$ with a small positive $\epsilon$. In so doing, the integral is just the residue at $\zeta=0$. To be more explicit, since $\zeta=0$ is a pole of order $(M+1)$, we have

$$
\begin{aligned}
\eta_{M}^{( \pm)}(z) & =\left.\frac{2 \pi}{M !}\left[\frac{d^{M}}{d \zeta^{M}} \exp \left\{ \pm i k_{c}(\zeta) z\right\}\right]\right|_{\zeta=0} \\
& =\left.\frac{2 \pi(-1)^{M}}{M !}\left[\frac{d^{M}}{d \zeta_{1}^{M}} \exp \left\{ \pm i k_{c}\left(\zeta_{1}\right) z\right\}\right]\right|_{\zeta_{1}=1},
\end{aligned}
$$

where we have introduced $\zeta_{1}=1-\zeta$, since $k_{c}(\zeta)$ is indeed a function of $1-\zeta$.

\section{B. Injection error}

For the case of injection error, the general solution is given in Eq. (67), i.e., for $M \neq 0$,

$$
\begin{aligned}
y_{M}(z) & =\frac{1}{2 \pi i} y_{00} \oint d \zeta \zeta^{-(M+1)}(1-\zeta)^{-1} \cos \left\{k_{c}(\zeta) z\right\} \\
& \equiv \frac{y_{00}}{4 \pi}\left[\eta_{M}^{(+)}(z)+\eta_{M}^{(-)}(z)\right]
\end{aligned}
$$

Similarly, this time we write

$$
\begin{aligned}
\eta_{M}^{( \pm)}(z) & \equiv \frac{1}{i} \oint d \zeta \zeta^{-(M+1)}(1-\zeta)^{-1} \exp \left\{ \pm i k_{c}(\zeta) z\right\} \\
& =\left.\frac{2 \pi}{M !}\left\{\frac{d^{M}}{d \zeta^{M}}\left[\frac{\exp \left\{ \pm i k_{c}(\zeta) z\right\}}{1-\zeta}\right]\right\}\right|_{\zeta=0} \\
& =\left.\frac{2 \pi(-1)^{M}}{M !}\left\{\frac{d^{M}}{d \zeta_{1}^{M}}\left[\zeta_{1}^{-1} \exp \left\{ \pm i k_{c}\left(\zeta_{1}\right) z\right\}\right]\right\}\right|_{\zeta_{1}=1}
\end{aligned}
$$

Equations (73) and (74) are now ready for direct numerical calculation, and the results could be used to check the analytical expression in Eqs. (42), (45), (69), and (71).

Before we go into detail of the calculation, let us make some comments. First, according to the general solution in Eq. (17), and the focusing strength in the NF limit given in Eq. (26), we conclude that the solution is a universal function of $a_{1} z$, hence, the result stays the same as long as the product $a_{1} z$ stays constant. In the SF limit, then according to Eq. (17) and the focusing strength in Eq. (27), the independent variables are $k_{y} z$ 
and $a_{2} z$. Second, according to the solutions given in Eqs. (42), (45), (69), and (71), the results depend only on the ratio of $t / t_{\mathrm{NF}}$ and $t / t_{\mathrm{SF}}$. We know that these are the long-time asymptotic behaviors, hence, in the numerical calculation, we only need calculate once according to the universal variable $t / t_{\mathrm{NF}}$ or $t / t_{\mathrm{SF}}$, and then rescale various cases according to their own $t_{\mathrm{NF}}$ and $t_{\mathrm{SF}}$. So, in the following, we will do calculations for some arbitrary chosen parameters, but the conclusion drawn from these examples is universal, based on the above analysis.

In our calculation for the NF limit, we choose $a=$ $0.5 \mathrm{~m}^{-2}, z=1.8 \mathrm{~m}$, this yields $t_{\mathrm{NF}} / \tau_{B} \approx 0.01$ for the case of only a single bunch is initially offset. While for the injection error case, we choose $a=0.05 \mathrm{~m}^{-2}, z=$ $1.8 \mathrm{~m}$, this yields $t_{\mathrm{NF}} / \tau_{B} \approx 1.0$. We further introduce the relative error as

$$
\delta_{M}(z) \equiv \frac{y_{M}^{\mathrm{Num}}(z)-y_{M}^{\mathrm{Asy}}(z)}{y_{M}^{\mathrm{Num}}(z)},
$$

where $y_{M}^{\mathrm{Num}}(z)$ is the numerical result given by Eq. (73) or Eq. (74), and $y_{M}^{\mathrm{Asy}}(z)$ is the asymptotic result given by Eq. (42) or Eq. (69) for the case of single-bunch offset or the injection error, respectively.

The results are given in Fig. 3. As we find, the asymptotic result agrees with the numerical result within $20 \%$ for bunch number $M>5$ and $M>1$ for the case of single-bunch offset and injection error, respectively. Recall that we set $t_{\mathrm{NF}} / \tau_{B} \approx 0.01$ and 1 for the case of single-bunch offset and injection error, respectively. Hence, the general conclusion will be the following: our asymptotic results given in Eqs. (42) and (69) agree with the numerical solution within $20 \%$, when $t>500 t_{\mathrm{NF}}$ and $t>t_{\mathrm{NF}}$ for the case of single-bunch offset and injection error, respectively. Shown in Fig. 3, the relative error is

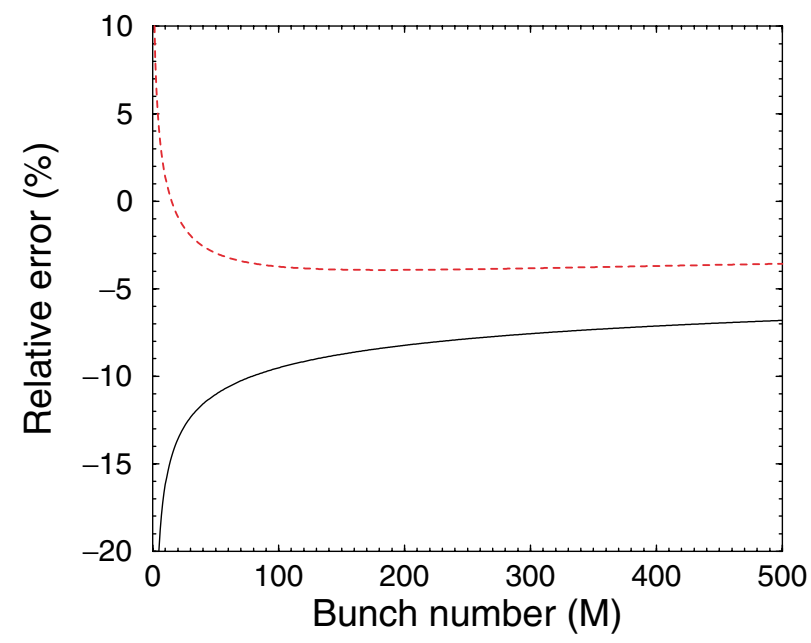

FIG. 3. (Color) The relative error defined in Eq. (75) as a function of the bunch number $M=t / \tau_{B}$. The solid curve stands for the case of single-bunch offset and the dashed curve for the case of an injection error.

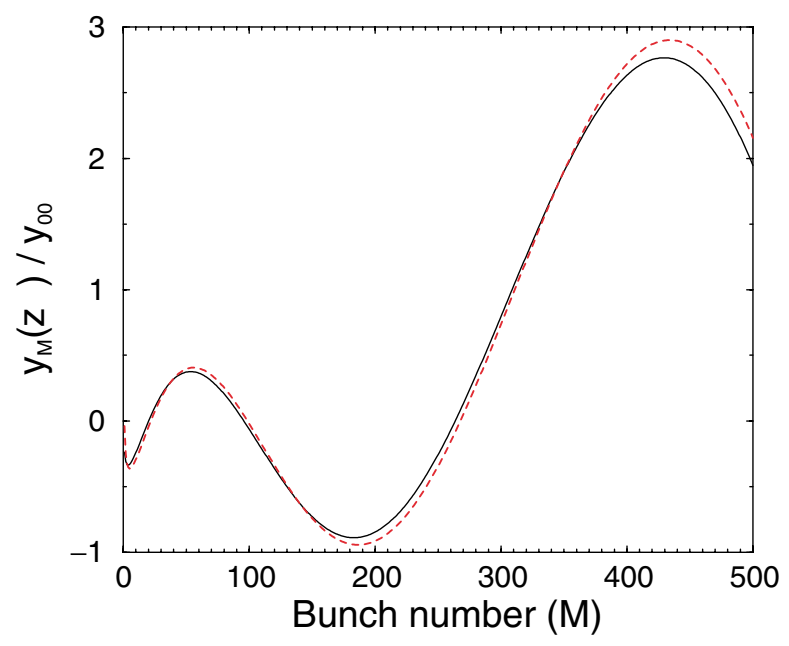

FIG. 4. (Color) The ratio $y_{M}(z) / y_{00}$ for the case of single-bunch offset as a function of the bunch number $M=t / \tau_{B}$. The solid curve stands for the result obtained numerically from Eq. (73) and the dashed curve for the asymptotic result from Eq. (45).

only a few percent when $M>80$ (i.e., $t>8000 t_{\mathrm{NF}}$ ) and $M>2$ (i.e., $t>2 t_{\mathrm{NF}}$ ) for the single-bunch offset and the injection error case and monotonically decreasing beyond that. This gives the general conclusion of how good the agreement is between the asymptotic analytical expression and the direct numerical solution. In real projects, $a$ and $z$ will vary from case to case, which result in various $t_{\mathrm{NF}}$, but the statement stays true with respect to $t_{\mathrm{NF}}$.

For the SF limit, we choose $a=1.46 \mathrm{~m}^{-2}, z=10 \mathrm{~m}$, and $k_{y}=12 \mathrm{~m}^{-1}$, and we have $t_{\mathrm{SF}} / \tau_{B} \approx 1$. Since the results are oscillatory solutions, a relative error as in Eq. (75) is not very instructive. Every time when $y_{M}^{\text {Asy }}(z)=0$, we would get $100 \%$ relative error which is really misleading. Hence, we instead plot $y_{M}(z) / y_{00}$ as a function of bunch number $M=t / \tau_{B}$.

The results are given in Figs. 4 and 5 for the case of single-bunch offset and injection error, respectively. As we find, as long as $t>t_{\mathrm{SF}}$, the asymptotic results in Eqs. (45) and (71) agree well with the numerical results obtained from Eqs. (73) and (74). Recall that in our calculation, we have set $t_{\mathrm{SF}} \approx \tau_{B}$. In Fig. 4 , there exists discrepancy between the result of the asymptotic analytical expression and that of the direct numerical solution around the turning points of the two curves at $M \sim 190$ (i.e., $t \sim 190 t_{\mathrm{SF}}$ ) and $M \sim 450$ (i.e., $t \sim 450 t_{\mathrm{SF}}$ ). However, the relative error decreases with $M$ (i.e., $t / t_{\mathrm{SF}}$ ) increases. Again, this general statement stays true with respect to $t_{\mathrm{SF}}$, though in real projects, $a, z$, and $k_{y}$ will vary from case to case, which results in various $t_{\mathrm{SF}}$.

\section{APPLICATION AND DISCUSSION}

Now let us study the projects we mentioned in Sec. I. For the PERL project, we hope to know whether the PERL beam can survive the entire injection period of 


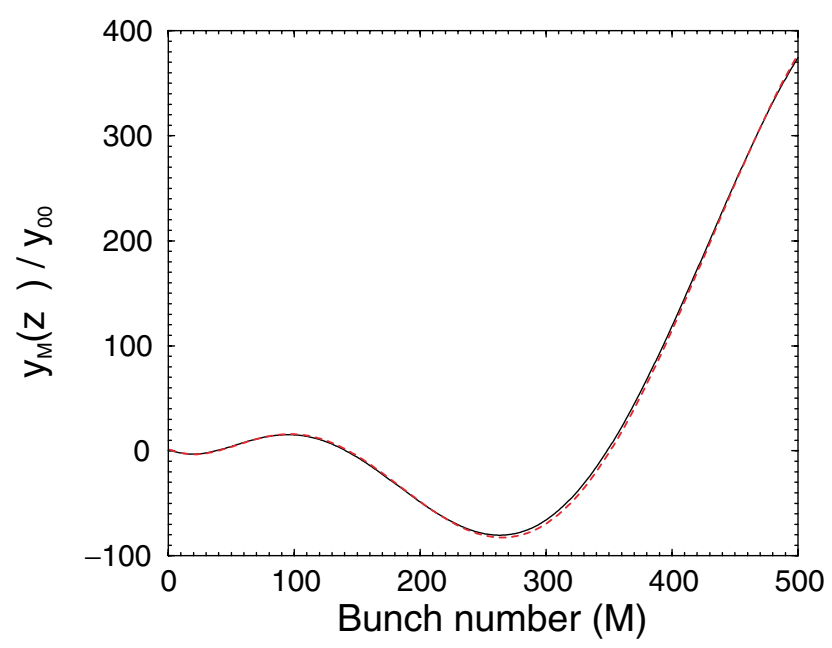

FIG. 5. (Color) The ratio $y_{M}(z) / y_{00}$ for the case of injection error as a function of the bunch number $M=t / \tau_{B}$. The solid curve stands for the result obtained numerically from Eq. (74) and the dashed curve for the asymptotic result from Eq. (71).

$12 \mathrm{~h}$. A preliminary parameters list of the PERL beam is given in Table I. For simplicity, we assume that there are no gaps between the insertion devices; in other words, we approximate the 12 insertion devices with gaps in between by a continuous pipe of $144 \mathrm{~m}$. The parameters of the other projects are also given in Table I.

For convenience, let us introduce

$$
\chi_{\mathrm{NF}} \equiv\left|a \sqrt{\pi /\left(1-\zeta_{\mathrm{NF}}\right)}\right| / k_{y}^{2}
$$

and

$$
\chi_{\mathrm{SF}} \equiv k_{y}^{2} /\left|a \sqrt{\pi /\left(1-\zeta_{\mathrm{SF}}\right)}\right|
$$

where $\zeta_{\mathrm{NF}}$ and $\zeta_{\mathrm{SF}}$ are the saddle points given in Eqs. (34) and (A5), respectively. For the NF limit to be applicable, we require $\chi_{\mathrm{NF}} \gg 1$, and for the SF limit, $\chi_{\mathrm{SF}} \gg 1$.

For the PERL project, if we use a conventional planar wiggler, then there is no horizontal focusing. Hence, the
NF limit is applied. Given the parameters in Table I but $k_{y}=0$, we get $t_{\mathrm{NF}} \approx 17 \mathrm{~ns}$, and the operation period of $12 \mathrm{~h}$ is much larger than $500 t_{\mathrm{NF}}$. Hence, according to the analysis in Sec. VII, we could safely use the asymptotic expressions in Eqs. (42) and (69). In the case of a single-bunch offset, we already get $y_{M}(z) / y_{00} \sim$ $1 \times 10^{69}$, so without focusing, the beam will not survive the entire injection period. We therefore assume that we add strong focusing in the horizontal plane to have $k_{y}=3 \mathrm{~m}^{-1}$. Given this, we find $t_{\mathrm{SF}} \approx 2 \mathrm{~s}$, and the injection period of $12 \mathrm{~h}$ is much larger than $t_{\mathrm{SF}}$. So according to the analysis in Sec. VII, the asymptotic expression is applicable. Next, $\chi_{\mathrm{NF}} \approx 0.1$ and $\chi_{\mathrm{SF}} \approx 6$, hence the SF limit is marginally applicable. We then apply the SF limit to get $y_{M}(z) / y_{00} \sim 1$ for the singlebunch offset case. While for the case of injection error, we have $y_{M}(z) / y_{00} \sim 2 \times 10^{11}$. Hence a feedback system is necessary, and should be easy to implement, since the growth time $t_{\mathrm{SF}} \approx 2 \mathrm{~s}$ is large enough. For the LCLS parameter, calculation shows that the beam can safely survive an assumed $12 \mathrm{~h}$ injection period, even with injection error. The very reason is the low rep rate, though other parameters are similar to the PERL project. A similar conclusion is found for the existing TTF VUV FEL. Because of the very low rep rate, even if the undulator is up to kilometers long, the effect is still negligible. For the NLC project, in the final focusing section, the averaged $\beta$ function is about $50 \mathrm{~km}$, which introduces negligible betatron phase advance during the $300 \mathrm{~m}$ long section, hence we take the NF limit. We investigate a train of 192 bunches with bunch separation of $1.4 \mathrm{~ns}$. Calculation shows that the cumulative resistive-wall effect is small. This time, it is because of the large beam pipe radius, since both $t_{\mathrm{NF}}$ and $t_{\mathrm{SF}} \propto b^{6}$. Also, this is only a single pass train of 192 bunches. Similar results are obtained for the FFS of TESLA, where we investigate a train of 2820 bunches with bunch separation of $337 \mathrm{~ns}$. The results are summarized in Table I.

TABLE I. Summary of the parameters and results for the PERL project, the LCLS project, the existing TTF VUV FEL, the FFS of the NLC project and the FFS of the TESLA project. "NG" in the table stands for negligible growth.

\begin{tabular}{lccccc}
\hline \hline & PERL & LCLS & TTF VUV FEL & NLC & TESLA \\
\hline Rep rate $f_{B}(\mathrm{GHz})$ & 1.3 & $1.2 \times 10^{-7}$ & $7 \times 10^{-8}$ & 0.714 & $2.97 \times 10^{-3}$ \\
Pipe radius $b(\mathrm{~cm})$ & 0.3 & 0.3 & 0.495 & 2 & 2 \\
Pipe length $(\mathrm{m})$ & 144 & 150 & 13.5 & 300 & 700 \\
Conductivity $\sigma_{c}\left(10^{7} \Omega^{-1} \mathrm{~m}^{-1}\right)$ & 6 & 6 & 3.47 & 3.47 & 3.47 \\
$k_{y}\left(\mathrm{~m}^{-1}\right)$ & 3 & $1 / 18$ & 1 & 0 & 0 \\
Beam energy $(\mathrm{GeV})$ & 3 & 14.35 & 0.24 & 250 & 250 \\
Bunch charge $(\mathrm{nC})$ & 0.15 & 1 & 2.8 & 1.2 & 3.2 \\
$t=M \tau_{B}$ & $12 \mathrm{~h}$ & $12 \mathrm{~h}$ & $12 \mathrm{~h}$ & $267.4 \mathrm{~ns}$ & $0.95 \mathrm{~ms}$ \\
$y_{M}(z) / y_{00}$ : single-bunch offset & $\mathrm{NG}$ & $\mathrm{NG}$ & $\mathrm{NG}$ & $\mathrm{NG}$ & $\mathrm{NG}$ \\
$y_{M}(z) / y_{00}$ : injection error & $2 \times 10^{11}$ & $\mathrm{NG}$ & $\mathrm{NG}$ & $\mathrm{NG}$ & $\mathrm{NG}$ \\
\hline \hline
\end{tabular}


In our calculation, we neglect the single-bunch effect, which is treated separately $[12,13]$.

\section{ACKNOWLEDGMENTS}

The authors thank Professor T. O. Raubenheimer of the Stanford Linear Accelerator Center (SLAC) for an illuminating comment. The authors also thank K. L. F. Bane of SLAC for reading the manuscript and for his interesting comments. This work was supported by the U.S. Department of Energy under Contracts No. DE-AC0298CH10886 (J.-M.W.) and No. DE-AC03-76SF00515 (J.W.).

\section{APPENDIX A: DETAILS FOR DERIVING EQ. (45)}

For the SF case, the exponent of the integrand in Eq. (22) is

$$
\Psi_{M}^{( \pm)}(\zeta)= \pm i k_{y} z \mp i 2 a_{2} z(1-\zeta)^{-1 / 2}-(M+1) \log (\zeta) .
$$

This function has branch points at $\zeta=0$ and $\zeta=1$. We cut the complex $\zeta$ plane from $\zeta=-\infty$ to 0 , and from $\zeta=$ 1 to $\infty$. The same contour is chosen as in Fig. 2. The first two derivatives of $\Psi_{M}^{( \pm)}(\zeta)$ are

$$
\dot{\Psi}_{M}^{( \pm)}(\zeta)=\mp i a_{2} z(1-\zeta)^{-3 / 2}-\frac{M+1}{\zeta},
$$

and

$$
\ddot{\Psi}_{M}^{( \pm)}(\zeta)=\mp i \frac{3}{2} a_{2} z(1-\zeta)^{-5 / 2}+\frac{M+1}{\zeta^{2}} .
$$

The saddle point condition $\dot{\Psi}_{M}^{( \pm)}\left(\zeta_{\mathrm{SF}}\right)=0$ leads to

$$
\left(1-\zeta_{\mathrm{SF}}\right)^{3 / 2}=\mp i \alpha_{2} \zeta_{\mathrm{SF}}
$$

with $\alpha_{2} \equiv a_{2} z /(M+1)$. Since $\alpha_{2} \rightarrow 0$, as $M \rightarrow \infty$, we could again find the saddle points by a perturbation method. The result is, to the leading order of $\alpha_{2}$,

$$
\zeta_{\mathrm{SF}}=\left(1-\alpha_{2}^{2 / 3} e^{i \pi / 3}, 1+\alpha_{2}^{2 / 3}, 1-\alpha_{2}^{2 / 3} e^{-i \pi / 3}\right),
$$

where we write the solutions of Eq. (A4) as elements of a $1 \times 3$ row matrix.

The Eq. (A5) is a necessary but not a sufficient condition for the saddle points. Simple algebraic calculations show that the first element of the matrix (A5) is a saddle point of $\eta_{M}^{(-)}$, and that the third element is a saddle point of $\eta_{M}^{(+)}$. The second element of (A5) which is $>1$ and lies on the branch cut is not accessible to the integration contour.

We need to evaluate $\Psi_{M}^{( \pm)}$and $\ddot{\Psi}_{M}^{( \pm)}$at the appropriate saddle points. They are

$$
\Psi_{M}^{(+)}\left(\zeta_{\mathrm{SF}, 3}\right)=+i k_{y} z+3(M+1) \alpha_{2}^{2 / 3} \exp \left\{-\frac{i \pi}{3}\right\},
$$

$$
\begin{gathered}
\ddot{\Psi}_{M}^{(+)}\left(\zeta_{\mathrm{SF}, 3}\right)=\frac{3(M+1)}{2 \alpha_{2}^{2 / 3}} \exp \left\{\frac{i \pi}{3}\right\}, \\
\Psi_{M}^{(-)}\left(\zeta_{\mathrm{SF}, 1}\right)=-i k_{y} z+3(M+1) \alpha_{2}^{2 / 3} \exp \left\{\frac{i \pi}{3}\right\},
\end{gathered}
$$

and

$$
\ddot{\Psi}_{M}^{(-)}\left(\zeta_{\mathrm{SF}, 1}\right)=\frac{3(M+1)}{2 \alpha_{2}^{2 / 3}} \exp \left\{-\frac{i \pi}{3}\right\} .
$$

Using these results, we obtain the asymptotic expression in Eq. (45).

\section{APPENDIX B: DETAILS FOR DERIVING EQ. (69)}

We carry out the saddle point analysis to the integral (22) with the exponent

$$
\begin{aligned}
\Psi_{M}^{( \pm)}(\zeta)= & \mp a_{1} z(1-\zeta)^{-1 / 4} \\
& -(M+1) \log (\zeta)-\log (1-\zeta) .
\end{aligned}
$$

The first two derivatives of the exponent are

$$
\dot{\Psi}_{M}^{( \pm)}(\zeta)=\mp \frac{1}{4} a_{1} z(1-\zeta)^{-5 / 4}-\frac{M+1}{\zeta}+\frac{1}{1-\zeta},
$$

and

$$
\ddot{\Psi}_{M}^{( \pm)}(\zeta)=\mp \frac{5}{16} a_{1} z(1-\zeta)^{-9 / 4}+\frac{M+1}{\zeta^{2}}+\frac{1}{(1-\zeta)^{2}} .
$$

The saddle points are determined by $\dot{\Psi}_{M}^{( \pm)}\left(\zeta_{\mathrm{NF}}\right)=0$, i.e.,

$$
0=\mp \frac{1}{4} a_{1} z\left(1-\zeta_{\mathrm{NF}}\right)^{-5 / 4}-\frac{M+1}{\zeta_{\mathrm{NF}}}+\frac{1}{1-\zeta_{\mathrm{NF}}},
$$

which cannot be solved algebraically. However, since the saddle points $\zeta_{\text {saddle }} \rightarrow 1$ in the limit of $M \rightarrow \infty$, we could solve Eq. (B4) by a perturbation method. In terms of $\tilde{\zeta} \equiv$ $1-\zeta$, Eq. (B4) becomes

$$
\mp \frac{1}{4} a_{1} z \tilde{\zeta}_{\mathrm{NF}}^{-5 / 4} \pm \frac{1}{4} a_{1} z \tilde{\zeta}_{\mathrm{NF}}^{-1 / 4}+\tilde{\zeta}_{\mathrm{NF}}^{-1}-1=M+1 .
$$

Keeping the leading term in Eq. (B5), we get

$$
\mp \frac{1}{4} a_{1} z \tilde{\zeta}_{\mathrm{NF}}^{-5 / 4}=M+1 .
$$

The last equation is identical to Eq. (32), and therefore it yields the same first-order solution given in Eq. (34). In other words, the positions of the saddle points are independent of the initial condition. We select now the relevant saddle points by repeating what we did before following Eq. (34), and then carry out the saddle point integral corresponding to the exponent (B1). We finally reach the expression in Eq. (69).

[1] R. L. Gluckstern, R. K. Cooper, and P. J. Channell, Part. Accel. 16, 125 (1985). 
[2] G. Decker and J. M. Wang, Phys. Rev. D 38, 980 (1988).

[3] J. J. Bisognano and R. L. Gluckstern, in Proceedings of the 1987 IEEE Particle Accelerator Conference (IEEE, Piscataway, NJ, 1988), p. 1078; G. A. Krafft and J. J. Bisognano, ibid., p. 1356.

[4] J. R. Delayen, Phys. Rev. ST Accel. Beams 6, 084402 (2003).

[5] G. J. Caporaso, W. A. Barletta, and V. K. Neil, Part. Accel. 11, 71 (1980).

[6] I. Ben-Zvi et al., in Proceedings of the 2001 IEEE Particle Accelerator Conference (IEEE, Piscataway, NJ, 2002), p. 350.

[7] J. Arthur et al., SLAC Report No. SLAC-R-593, 2002.

[8] J. Andruszkov et al., Phys. Rev. Lett. 85, 3825 (2000).

[9] T. O. Raubenheimer, in Progress in the Next Linear Collider Design, AIP Conf. Proc. No. 578 (AIP, New York, 2001), pp. 53-64; NLC ZDR Design Group, SLAC Report No. 474, 1996.

[10] "TESLA: The Superconducting Electron-Positron Linear Collider with an Integrated X-Ray Laser Laboratory," DESY Technical Design Report, 2001.

[11] P. L. Morton, V. K. Neil, and A. M. Sessler, J. Appl. Phys. 37, 3875 (1966).
[12] J.-M. Wang, S. R. Mane, and N. Towne, in Proceedings of the 7th European Particle Accelerator Conference, EPAC'00, Vienna, Austria (CERN, Geneva, 2000), p. 1179.

[13] J. R. Delayen, T. O. Raubenheimer, J.-M. Wang, and J. Wu (to be published).

[14] This method of relating the transient BBU problem to the coherent solution of the "extended problem" has previously been used in Ref. [2] and C. L. Bohn and J. R. Delayen, Phys. Rev. A 45, 5964 (1992). The appendix of [2] is devoted to a detailed explanation of the meaning of the extended problem.

[15] E. D. Courant and A. M. Sessler, Rev. Sci. Instrum. 37, 1579 (1966)

[16] A summary of the properties of the function $\sum_{m=0}^{\infty} \zeta^{m} / \sqrt{m+v}$ can be found in Higher Transcendental Functions, edited by A. Erdèlyi (Krieger Publishing Co., Malabar, Florida, 1981), Vol. I, Sect. 1.11.

[17] J.-M. Wang and J. Wu, in Proceedings of the 8th European Particle Accelerator Conference, EPAC'02, Paris, France (EPS-IGA/CERN, Geneva, 2002), p. 1580. 